\title{
МАТЕМАТИКА
}

\section{P.V. Danchev}

\section{COMMUTATIVE FEEBLY INVO-CLEAN GROUP RINGS}

\begin{abstract}
A commutative ring $R$ is called feebly invo-clean if any its element is of the form $v+e-f$, where $v$ is an involution and $e, f$ are idempotents. For every commutative unital ring $R$ and every abelian group $G$ we find a necessary and sufficient condition only in terms of $R, G$ and their sections when the group ring $R[G]$ is feebly invo-clean. Our result improves two recent own achievements about commutative invo-clean and weakly invo-clean group rings, published in Univ. J. Math. \& Math. Sci. (2018) and Ural Math. J. (2019), respectively.
\end{abstract}

Keywords: invo-clean rings, weakly invo-clean rings, feebly invo-clean rings, group rings.

\section{Introduction and Conventions}

Throughout the current paper, we will assume that all groups $G$ are multiplicative abelian and all rings $R$ with Jacobson radical $J(R)$ are associative, containing the identity element 1 which differs from the zero element 0 . The standard terminology and notation are mainly in agreement with [9 and 10], whereas the specific notion and notation shall be explained explicitly below. As usual, both objects $R$ and $G$ form the group ring $R[G]$ of $G$ over $R$.

The next concepts appeared in [1, 2, and 3], respectively.

Definition 1.1. A ring $R$ is said to be invo-clean if, for each $r \in R$, there exist an involution $v$ and an idempotent $e$ such that $r=v+e$. If $r=v+e$ or $r=v-e$, the ring is called weakly invo-clean.

The next necessary and sufficient condition for a commutative ring $R$ to be invoclean was established in [1,2], namely: $A$ ring $R$ is invo-clean if, and only if, $R \cong R_{1} \times R_{2}$, where $R_{1}$ is a nil-clean ring with $z^{2}=2 z$ for all $z \in J\left(R_{1}\right)$, and $R_{2}$ is a ring of characteristic 3 whose elements satisfy the equation $x^{3}=x$. Moreover, it was proved in [6] that a ring $R$ is weakly invo-clean $\Leftrightarrow$ either $R$ is invo-clean or $R$ can be decomposed as $R=K \times \mathbb{Z}_{5}$, where $K=\{0\}$ or $K$ is invo-clean.

The above two notions could be expanded as follows:

Definition 1.2. A ring $R$ is said to be feebly invo-clean if, for each $r \in R$, there exist an involution $v$ and idempotents $e, f$ such that $r=v+e-f$.

We will give up in the sequel an useful criterion for a commutative ring to be feebly invo-clean in order to be successfully applied to commutative group rings (compare with Proposition 2.2). 
It was asked in [6] to find a suitable criterion only in terms of the commutative unital ring $R$ and the abelian group $G$ when the group ring $R[G]$ is feebly invo-clean. So, the goal of this short article is to address that question in the affirmative. Some related results in this area can also be found in [4 and 7].

\section{The Characterization Result}

We begin here with the following key formula from [8] which will be freely used below without concrete citation: Suppose that $R$ is a commutative ring and $G$ is an abelian group. Then

$$
J(R[G])=J(R)[G]+\left\langle r(g-1) \mid g \in G_{p}, p r \in J(R)\right\rangle,
$$

where $G_{p}$ designates the $p$-primary component of $G$.

The next two technicalities are crucial for our further considerations.

Lemma 2.1. Let $K$ be a commutative ring of characteristic 5. Then $K$ is feebly invo-clean $\Leftrightarrow x^{5}=x$ holds for any $x \in K$.

Proof. The "left-to-right" implication is almost trivial as writing $x=v+e-f$ with $v^{2}=1, e^{2}=e$ and $f^{2}=f$, we have that $x^{5}=(v+e-f)^{5}=v^{5}+e^{5}-f^{5}=v+e-f=x$, as asserted.

As for the "right-to-left" implication, we process like this: Given an arbitrary nonidentity element $x$ in $K$. Then the subring, $S$, generated by 1 and $x$ will have the same property, namely its characteristic is again 5 and $y^{5}=y$ for all $y \in S$. So, with no harm of generality, we may replace $K$ by this subring $S$, and thus it needs to prove the wanted representation property in $S$ only. To that purpose, we claim that $S$ is isomorphic to a quotient of the factor-ring $\mathbb{Z}_{5}[X] /\left(X^{5}-X\right) \cong \mathbb{Z}_{5} \times \mathbb{Z}_{5} \times \mathbb{Z}_{5} \times \mathbb{Z}_{5} \times \mathbb{Z}_{5}$ of the polynomial ring $\mathbb{Z}_{5}[X]$ over $\mathbb{Z}_{5}$. In fact, we just consider the map $\mathbb{Z}_{5}[X] \rightarrow S$, defined by mapping $X \rightarrow x$, which is elementary checked to be a surjective homomorphism with kernel which contains the ideal generated by $X^{5}-X$, and henceforth the classical Homomorphism Theorem works to get the desired claim. Working now in the direct product of five copies of the five-element field $\mathbb{Z}_{5}=\{0,1,2,3,4 \mid 5=0\}$, a plain technical argument gives our wanted initial assertion that $S$ and hence $K$ are both feebly invo-clean. This is subsumed by the presentations $0=1+0-1,1=1+0-0$, $2=1+1-0,3=4+0-1$ and $4=4+0-0$, where $4^{2}=1,1^{2}=1$ and $0^{2}=0$.

Proposition 2.2. A commutative ring $R$ is feebly invo-clean $\Leftrightarrow R=P \times K$ for two rings $P, K$, where $P=\{0\}$ or $P$ is invo-clean, and $K=\{0\}$ or $K$ possesses characteristic 5 such that $x^{5}=x, \forall x \in K$.

Proof. $" \Rightarrow "$. It follows from the corresponding characterization method used in [3, Theorem 2.6].

$" \Leftarrow "$. Firstly, it needs to show that $K$ is feebly invo-clean. This, however, follows directly from Lemma 2.1. Furthermore, one suffices to observe again with [3, Theorem 2.6] at hand that the direct product of such a ring $K$ with an invo-clean ring remains a feebly invo-clean ring, thus getting resultantly that $R$ is feebly invo-clean, as expected.

We are now ready to proceed by proving the following preliminary statement (see [5] as well). 
Proposition 2.3. Suppose $R$ is a non-zero commutative ring and $G$ is an abelian group. Then $R[G]$ is invo-clean if, and only if, $R$ is invo-clean having the decomposition $R=R_{1} \times R_{2}$ such that precisely one of the next three items holds:

(0) $G=\{1\}$

or

(1) $|G|>2, G^{2}=\{1\}, R_{1}=\{0\}$ or $R_{1}$ is a ring of char $\left(R_{1}\right)=2$, and $R_{2}=\{0\}$, or $R_{2}$ is a ring of char $\left(R_{2}\right)=3$

or

(2) $|G|=2,2 r_{1}^{2}=2 r_{1}$ for all $r_{1} \in R_{1}$ (in addition $4=0$ in $R_{1}$ ), and $R_{2}=\{0\}$ or $R_{2}$ is a ring of char $\left(R_{2}\right)=3$.

Proof. If $G$ is the trivial i.e., the identity group, there is nothing to do, so we shall assume hereafter that $G$ is non-identity.

"Necessity." Since there is an epimorphism $R[G] \rightarrow R$, and an epimorphic image of an invo-clean ring is obviously an invo-clean ring (see, e.g., [1]), it follows at once that $R$ is again an invo-clean ring. According to the criterion for invo-cleanness alluded to above, one writes that $R=R_{1} \times R_{2}$, where $R_{1}$ is a nil-clean ring with $a^{2}=2 a$ for all $a \in J\left(R_{1}\right)$ and $R_{2}$ is a ring whose elements satisfy the equation $x^{3}=x$. Therefore, it must be that $R[G] \cong R_{1}[G] \times R_{2}[G]$, where it is not too hard to verify by [1] that both $R_{1}[G]$ and $R_{2}[G]$ are invo-clean rings.

First, we shall deal with the second direct factor $R_{2}[G]$ being invo-clean. Since $\operatorname{char}\left(R_{2}\right)=3$, it follows immediately that $\operatorname{char}\left(R_{2}[G]\right)=3$ too. Thus an application of an assemble of facts from $[1,2]$ allows us to deduce that all elements in $R_{2}[G]$ also satisfy the equation $y^{3}=y$. So, given $g \in G \subseteq R[G]$, it follows that $g^{3}=g$, that is, $g^{2}=1$.

Next, we shall treat the invo-cleanness of the group ring $R_{1}[G]$. Since char $\left(R_{1}\right)$ is a power of 2 (see [1]), it follows the same for $R_{1}[G]$. Consequently, utilizing once again an assortment of results from $[1,2]$, we infer that $R_{1}[G]$ should be nil-clean, so that $z^{2}=2 z$ for all $z \in J\left(R_{1}[G]\right)$. That is why, invoking the criterion from [7], we have that $G$ is a 2 -group. We claim that even $G^{2}=1$. In fact, for an arbitrary $g \in G$, we derive with the aid of the aforementioned formula from [8] that $1-g \in J\left(R_{1}[G]\right)$, because $2 \in J\left(R_{1}\right)$. Hence $(1-g)^{2}=2(1-g)$ which forces that $1-2 g+g^{2}=2-2 g$ and that $g^{2}=1$, as desired. We now assert that $\operatorname{char}\left(R_{1}\right)=2$ whenever $|G|>2$. To that purpose, there are two nonidentity elements $g \neq h$ in $G$ with $g^{2}=h^{2}=1$. Furthermore, again appealing to the formula from [8], the element $1-g+1-h=2-g-h$ lies in $J\left(R_{1}[G]\right)$, because $2 \in J\left(R_{1}\right)$. Thus $(2-g-h)^{2}=2(2-g-h)$ which yields that $2-2 g-2 h+2 g h=0$. Since $g h \neq 1$ as for otherwise $g=h^{-1}=h$, a contradiction, this record is in canonical form. This assures that $2=0$, as wanted. 
However, in the case when $|G|=2$, i.e. when $G=\left\{1, g \mid g^{2}=1\right\}=\langle g\rangle$, we can conclude that $2 r^{2}=2 r$ for any $r \in R_{1}$. Indeed, in view of the already cited formula from [8], the element $r(1-g)$ will always lie in $J\left(R_{1}[G]\right)$, because $2 \in J\left(R_{1}\right)$. We therefore may write $[r(1-g)]^{2}=2 r(1-g)$ which ensures that $2 r^{2}-2 r^{2} g=2 r-2 r g$ is canonically written on both sides. But this means that $2 r^{2}=2 r$, as pursued. Substituting $r=2$, one obtains that $4=0$. Notice also that $2 r^{2}=2 r$ for all $r \in R_{1}$ and $a^{2}=2 a$ for all $a \in J\left(R_{1}\right)$ will imply that $a^{2}=0$.

"Sufficiency." Foremost, assume that (1) is true. Since $R_{1}$ has characteristic 2 , whence it is nil-clean, and $G$ is a 2 -group, an appeal to [7] allows us to get that $R_{1}[G]$ is nil-clean as well. Since $z^{2}=2 z=0$ for every $z \in J\left(R_{1}\right)$, it is routinely checked that $\delta^{2}=2 \delta=0$ for each $\delta \in J\left(R_{1}[G]\right)$, exploiting the formula from [8] for $J\left(R_{1}[G]\right)$ and the fact that $R_{1}[G]$ is a modular group algebra of characteristic 2 . That is why, by a consultation with [1], one concludes that $R_{1}[G]$ is invo-clean, as expected. Further, by a new usage of [1], we derive that $R_{2}[G]$ is an invo-clean ring of characteristic 3 . To see that, given $x \in R_{2}[G]$, we write $x=\sum_{g \in G} r_{g} g$ with $r_{g} \in R_{2}$ satisfying $r_{g}^{3}=r_{g}$. Since $G^{2}=1$ will easily imply that $g^{3}=g$, one obtains that $x^{3}=\left(\sum_{g \in G} r_{g} g\right)^{3}=\sum_{g \in G} r_{g}^{3} g^{3}=\sum_{g \in G} r_{g} g=x$, as needed. We finally conclude with the help of [1] that $R[G] \cong R_{1}[G] \times R_{2}[G]$ is invo-clean, as expected.

Let us now point (2) be fulfilled. Since $G^{2}=1$, similarly to (1), $R_{2}$ being invoclean of characteristic 3 implies that $R_{2}[G]$ is invo-clean, too. In order to prove that $R_{1}[G]$ is invo-clean, we observe that $R_{1}$ is nil-clean with $2 \in J\left(R_{1}\right)$. According to [7], the group ring $R_{1}[G]$ is also nil-clean. What remains to show is that for any element $\delta$ of $J\left(R_{1}[G]\right)$ the equality $\delta^{2}=2 \delta$ is valid. Since in conjunction with the explicit formula quoted above for the Jacobson radical, an arbitrary element in $J\left(R_{1}[G]\right)$ has the form $j+j^{\prime} g+r(1-g)$, where $j, j^{\prime} \in J\left(R_{1}\right)$ and $r \in R_{1}$, we have that $\left[j+j^{\prime} g+r(1-g)\right]^{2} \in\left(J\left(R_{1}\right)^{2}+2 J\left(R_{1}\right)\right)[G]+r^{2}(1-g)^{2}$. However, using the given conditions, $z^{2}=2 z=2 z^{2}$ and thus $z^{2}=2 z=0$ for any $z \in J\left(R_{1}\right)$. Consequently, one checks that $\left[j+j^{\prime} g+r(1-g)\right]^{2}=r^{2}(1-g)^{2}=2 r^{2}(1-g)=2 r(1-g)=2\left[j+j^{\prime} g+r(1-g)\right]$, because $2 r^{2}=2 r$, as required. Therefore, $R_{1}[G]$ is invo-clean with [1] at hand. Finally, again [1] gives that $R[G] \cong R_{1}[G] \times R_{2}[G]$ is invo-clean, as promised.

It is worthwhile noticing that concrete examples of an invo-clean ring of characteristic 4 , such that its elements are solutions of the equation $2 r^{2}=2 r$, are the rings $\mathbb{Z}_{4}$ and $\mathbb{Z}_{4} \times \mathbb{Z}_{4}$. 
We thereby come to our main theorem which states the following:

Theorem 2.4. Let $G$ be an abelian group and let $R$ be a commutative non-zero ring. Then the group ring $R[G]$ is feebly invo-clean if, and only if, at most one of the next points is valid:

(1) $G=\{1\}$ and $R$ is feebly invo-clean.

(2) $G \neq\{1\}$ and $R \cong P \times K$, where $P \cong R_{1} \times R_{2}$ is an invo-clean ring and either $K=\{0\}$ or $K$ is a ring of char $(K)=5$ which is a subdirect product of a family of copies of the field $\mathbb{Z}_{5}$ such that either

(2.1) $P=\{0\}$ and $G^{4}=\{1\}$

or

(2.2) $|G|>2, G^{2}=\{1\}, P \neq\{0\}$ with $R_{1}=\{0\}$ or $R_{1}$ is a ring of char $\left(R_{1}\right)=2$ and $R_{2}=\{0\}$ or $R_{2}$ is a ring of char $\left(R_{2}\right)=3$

or

(2.3) $|G|=2, P \neq\{0\}$ with $2 r_{1}^{2}=2 r_{1}$ for all $r_{1} \in R_{1}$ (in addition $4=0$ in $R_{1}$ ) and $R_{2}=\{0\}$ or $R_{2}$ is a ring of char $\left(R_{2}\right)=3$.

Proof. If $G$ is trivial, there is nothing to prove because of the validity of the isomorphism $R[G] \cong R$, so let us assume hereafter that $G$ is non-trivial.

"Necessity." As the feebly invo-cleanness of the group ring $R[G]$ implies the same property for $R$, utilizing Proposition 2.2 we come to the fact that $R[G] \cong P[G] \times K[G]$ will imply feebly invo-cleanness of both group rings $P[G]$ and $K[G]$ whence $P[G]$ is necessarily invo-clean whereas $K[G]$ is either zero or a subdirect product of a family of copies of the field $\mathbb{Z}_{5}$. After that, under the presence of $P[G] \neq\{0\}$, we just need apply Proposition 2.3 to deduce the described above things in points (2), (2.2) and (2.3). Letting now $P[G]=\{0\}$, we shall deal only with $K[G]$. To that goal, what we now assert is that the group ring $K[G]$ having the property $x^{5}=x$ for all $x \in K[G]$ with $\operatorname{char}(K[G])=5$ yields that $K$ has the property $y^{5}=y$ for all $y \in K$ with $\operatorname{char}(K)=5$ and $G^{4}=\{1\}$. Indeed, since $K \subseteq K[G]$ and $G \subseteq K[G]$, this can be extracted elementarily thus substantiating our initial statement after all.

"Sufficiency." Item (2) ensures that $R[G] \cong P[G] \times K[G]$ and so it is simple verified that the feebly invo-cleanness of both $P[G]$ and $K[G]$ will assure feebly invocleanness of $R[G]$ as well. That is why, we will be concentrated separately on these two group rings. Firstly, the stated above conditions are a guarantor with the aid of Proposition 2.3 that $P[G]$ is invo-clean. Secondly, it is pretty easily seen that as $y^{5}=y$ and $g^{5}=g$ for all $y \in K$ and $g \in G$, because $K$ is a subdirect product of copies of the field $\mathbb{Z}_{5}$ possessing characteristic 5 and $G^{4}=\{1\}$, we may conclude that $x^{5}=x$ holds in $K[G]$ too, as required. This substantiates our former assertion after all. 


\section{REFERENCES}

1. Danchev P.V. (2017) Invo-clean unital rings. Commun. Korean Math. Soc. 32(1). pp. 19-27.

2. Danchev P.V. (2017) Weakly invo-clean unital rings. Afr. Mat. 28(7-8). pp. 1285-1295.

3. Danchev P.V. (2017) Feebly invo-clean unital rings. Ann. Univ. Sci. Budapest (Math.) 60. pp. 85-91.

4. Danchev P.V. (2017) Weakly semi-boolean unital rings. JP J. Algebra, Numb. Th. \& Appl. 39(3). pp. 261-276.

5. Danchev P.V. (2018) Commutative invo-clean group rings. Univ. J. Math. \& Math. Sci. 11(1). pp. 1-6.

6. Danchev P.V. (2019) Commutative weakly invo-clean group rings. Ural Math. J. 5(1). pp. $48-52$.

7. P.V. Danchev and W.Wm. McGovern (2015) Commutative weakly nil clean unital rings. J. Algebra. 425(5). pp. 410-422.

8. Karpilovsky G. (1982) The Jacobson radical of commutative group rings. Arch. Math. 39. pp. $428-430$.

9. Milies C.P. and Sehgal S.K. (2002) An Introduction to Group Rings. Vol. 1. Springer Science and Business Media.

10. Passman D. (2011) The Algebraic Structure of Group Rings. Dover Publications.

Received: June 4, 2019

DANCHEV Peter $V$. (Institute of Mathematics and Informatics, Bulgarian Academy of Sciences, Sofia, Bulgaria). E-mail: pvdanchev@yahoo.com, danchev@math.bas.bg

Данчев П.В. КОММУТАТИВНЫЕ МАЛО ИНВО-ЧИСТЫЕ ГРУППОВЫЕ КОЛЬЦА. Вестник Томского государственного университета.Математика и механика. 2019. № 61. C. $5-10$

\section{DOI $10.17223 / 19988621 / 61 / 1$}

Ключевые слова: инво-чистые кольца, слабо инво-чистые кольца, мало инво-чистые кольца, групповые кольца.

Коммутативное кольцо $R$ называется мало инво-чистым, если каждый его элемент имеет вид $v+e-f$, где $v$ - инволюция, а $e, f$ - идемпотенты. Для каждого коммутативного унитального кольца $R$ и каждой абелевой группы $\mathrm{G}$ найдены необходимые и достаточные условия, когда групповое кольцо $R[G]$ мало инво-чисто. Результаты статьи улучшают два последних достижения автора по коммутативным инво-чистым и слабо инво-чистым групповым кольцам, опубликованные в Универсальном журнале математики и математических наук (2018) и Уральском математическом журнале (2019) соответственно.

Danchev P.V. (2019) COMMUTATIVE FEEBLY INVO-CLEAN GROUP RINGS. Vestnik Tomskogo gosudarstvennogo universiteta. Matematika i mekhanika [Tomsk State University Journal of Mathematics and Mechanics]. 61.pp. 5-10

AMS Mathematical Subject Classification: 20C07; 16D60; 16S34; 16U60 